Kastrup, Julia; Steinke, Gorden

\title{
Essbiografisches Lernen mit minderjährigen Flüchtlingen in der
}

\section{Berufsvorbereitung}

Haushalt in Bildung \& Forschung 5 (2016) 1, S. 76-90

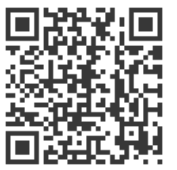

Quellenangabe/ Reference:

Kastrup, Julia; Steinke, Gorden: Essbiografisches Lernen mit minderjährigen Flüchtlingen in der

Berufsvorbereitung - In: Haushalt in Bildung \& Forschung 5 (2016) 1, S. 76-90 - URN:

urn:nbn:de:0111-pedocs-202778 - DOI: 10.25656/01:20277

https://nbn-resolving.org/urn:nbn:de:0111-pedocs-202778

https://doi.org/10.25656/01:20277

in Kooperation mit / in cooperation with:

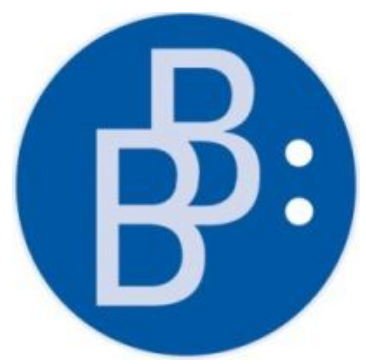

https://www.budrich.de

\section{Nutzungsbedingungen}

Gewährt wird ein nicht exklusives, nicht übertragbares, persönliches und beschränktes Recht auf Nutzung dieses Dokuments. Dieses Dokument ist ausschließlich für den persönlichen, nicht-kommerziellen Gebrauch bestimmt. Die Nutzung stellt keine Übertragung des Eigentumsrechts an diesem Dokument dar und gilt vorbehaltlich der folgenden Einschränkungen Auf sämtlichen Kopien dieses Dokuments müssen alle Urheberrechtshinweise und sonstigen Hinweise auf gesetzlichen Schutz beibehalten werden. Sie dürfen dieses Dokument nicht in irgendeiner Weise abändern, noch dürfen Sie dieses Dokument für öffentliche oder kommerzielle Zwecke vervielfältigen, öffentlich ausstellen, aufführen, vertreiben oder anderweitig nutzen.

Mit der Verwendung dieses Dokuments erkennen Sie die Nutzungsbedingungen an.

\section{Terms of use}

We grant a non-exclusive, non-transferable, individual and limited right to using this document

This document is solely intended for your personal, non-commercial use. Use of this document does not include any transfer of property rights and it is conditional to the following limitations: All of the copies of this documents must retain all copyright information and other information regarding legal protection. You are not allowed to alter this document in any way, to copy it for public or commercial purposes, to exhibit the document in public, to perform, distribute or otherwise use the document in public.

By using this particular document, you accept the above-stated conditions of use.

\section{Kontakt / Contact:}

\section{peDOcs}

DIPF | Leibniz-Institut für Bildungsforschung und Bildungsinformation Informationszentrum (IZ) Bildung

E-Mail:pedocs@dipf.de

Internet: www.pedocs.de

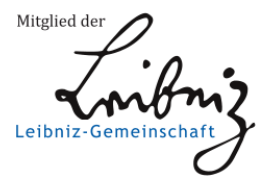


5. Jahrgang Heft 1 2016

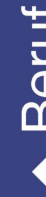

$\frac{1}{5}$

$\diamond$

(1)

(1)

ชิ

此

$\varepsilon$

ธิ

을

$\stackrel{\overline{1}}{>}$

$\bullet$

(1)

8

$\frac{1}{c}$

ब

$\varepsilon$

ह

क

N

$\bullet$

$\pm$

$\frac{1}{3}$
$\frac{3}{5}$
$+\frac{1}{0}$
$\frac{5}{0}$
$\frac{5}{5}$
0
0
0

ISSN 2193-8806

\section{Haushalt in}

Berufliche Bildung

Gestaltungsmöglichkeiten und

Zukunftsoptionen

IONDEILINL

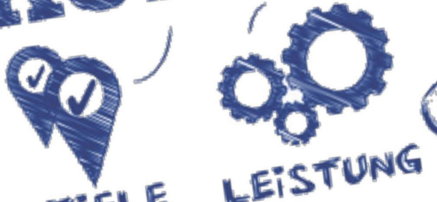

ZiEle leistung

FAHIGKEETEN
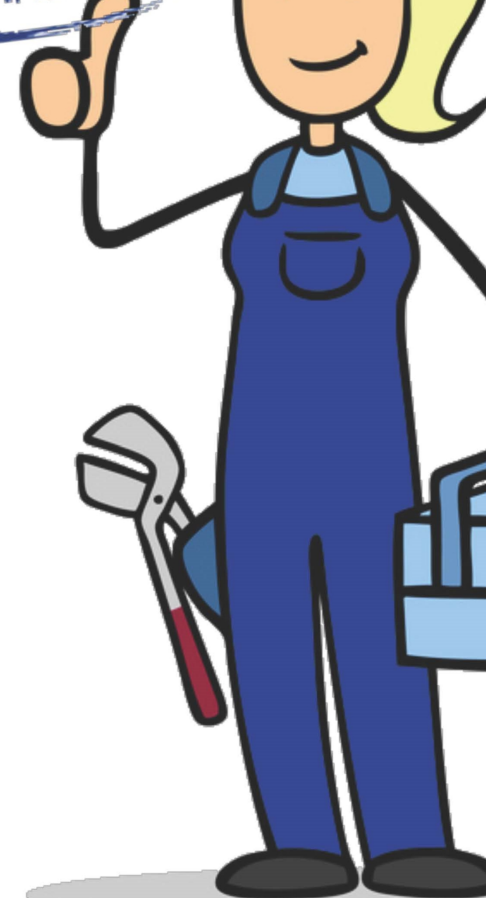
Inhaltsverzeichnis |

Julia Kastrup \& Irmhild Kettschau

Editorial.

Julia Kastrup \& Irmhild Kettschau

Standortentwicklungen, Nachwuchssituation und Nachwuchsförderung

in der beruflichen Fachdidaktik

Michael Martin

Der Berufsdidaktische Dreidecker.

Christine Küster, Nadine Harle \& Malte Matthias Flöper

Heterogenität als Zukunftsoption: Gestaltungsmöglichkeiten

des individualisierten Unterrichts in der beruflichen Bildung.

Anna Hoff

Interkulturelle Kompetenzen - Ein Blick auf die berufliche

Handlungsfähigkeit.

Julia Hirsch \& Nicole Fritsche

Marketing im Gastgewerbe - Von der Fachwissenschaft

zum beruflichen Lehramtsstudium

Julia Kastrup \& Gorden Steinke

Essbiografisches Lernen mit minderjährigen Flüchtlingen

in der Berufsorientierung.

Meike Ernestine Tecklenburg

Nachhaltiges Handeln in der Kita-Verpflegung:

Herausforderungen für die Weiterbildung.....

Marie Nölle

Nachhaltigkeitsbezogene Weiterbildungen im Berufsfeld

Ernährung und Gesundheit - ein Überblick zu Angebot und Bedarf 
Julia Kastrup \& Gorden Steinke

\section{Essbiografisches Lernen mit minderjährigen Flüchtlingen in der Berufsvorbereitung}

Eine Möglichkeit, minderjährige Flüchtlinge in die Gesellschaft $\mathrm{zu}$ integrieren, ist, ihnen berufliche Perspektiven aufzuzeigen. Das vorliegende didaktische Konzept verfolgt daher den Ansatz einer fächerübergreifenden Ernährungs- und Verbraucherbildung mit dem Ziel Handlungskompetenzen zu fördern. Durch die Verknüpfung von interkulturellem, essbiografischem und handlungsorientiertem Lernen steht, neben dem Spracherwerb und der Alltagsorientierung, eine gastgewerbliche Berufsfeldorientierung im Fokus des Konzeptes.

Schlüsselwörter: Essbiografisches Lernen, minderjährige Flüchtlinge, Berufsorientierung, handlungsorientiertes Lernen, interkulturelles Lernen

\section{Die Organisation der Berufsorientierung für minderjährige Flüchtlinge}

Schätzungen des Bundesfachverbandes „Unbegleitete Minderjährige Flüchtlinge e.V." gehen davon aus, dass im Jahr 201530.000 unbegleitete minderjährige Flüchtlinge nach Deutschland gekommen sind - doppelt so viele wie im vorangegangen Jahr. Die Gesamtzahl liegt damit momentan bei mehr als 45.000 Jugendlichen, die gemäß $\S 42$ Abs. 1 SGB VIII von deutschen Jugendämtern in Obhut genommen werden (Bundesfachverband UMF e.V., 2015). Hinzu kommen die minderjährigen Flüchtlinge in Begleitung, die jedoch von den Jugendämtern nicht systematisch erfasst werden.

Vor dem Hintergrund der weltweiten Krisenherde ist mit weiter steigenden Flüchtlingszahlen zu rechnen. Eine Herausforderung im Kontext dieser Entwicklung ist die nachhaltige Integration der Menschen in unsere Gesellschaft. Diese ist dann erfolgreich, wenn den Flüchtlingen eine Teilhabe an der Gesellschaft aus eigenen Mitteln möglich ist.

Eine Möglichkeit, die Integration der minderjährigen Flüchtlinge zu unterstützen, ist es, ihnen berufliche Perspektiven aufzuzeigen und sie langfristig in den Arbeitsmarkt zu integrieren. Dies ist auch vor dem Hintergrund des demografischen Wandels unserer Gesellschaft und dem damit verbundenen Fachkräftebedarf bedeutsam. Ebenso wie in gewerblich-technischen Berufsfeldern, besteht auch im Berufsfeld Ernährung und Hauswirtschaft ein akuter Fachkräftemangel (Helmrich 
et al., 2012, S. 9ff.). Durch eine entsprechende Berufsorientierung innerhalb der Berufsvorbereitung können den jungen Menschen/Migranten Möglichkeiten und Chancen einer Ausbildung und späteren beruflichen Tätigkeit in diesen Berufen aufgezeigt werden. Das bedeutet, dass durch eine gezielte Ausrichtung von Bildungsangeboten für minderjährige Flüchtlinge im Rahmen der Berufsvorbereitung einerseits eine realistische Möglichkeit zur Teilhabe am deutschen Arbeitsmarkt entwickelt wird, andererseits dem Fachkräftemangel begegnet werden kann. Ein hierfür erforderlicher arbeitsmarktintegrativer Bildungsansatz formuliert das Bildungsangebot von Anfang an berufsfeldorientiert und fördert mit geeigneten Methoden sowohl den Spracherwerb als auch erste berufsbezogene Kompetenzen.

Im vorliegenden Beitrag werden erste Umrisse für ein didaktisches Konzept skizziert, das die genannten Aspekte sowie die Besonderheiten der minderjährigen Flüchtlinge berücksichtigt.

Die Schulpflicht für neuankommende minderjährige Flüchtlinge ist in Deutschland über die landesrechtlichen Schulgesetze geregelt. Je nach Bundesland und abhängig vom aufenthaltsrechtlichen Status setzt die Schulpflicht bereits mit Ankunft der Flüchtlinge oder erst Monate später ein (Weiser, 2013, S. 8 ff.). Der Zugang zu Bildungsangeboten für nicht mehr schulpflichtige Flüchtlinge unterscheidet sich ebenfalls von Bundesland zu Bundesland, wobei keine Regelungen existieren, nach denen ein Berufsschulbesuch abhängig ist von aufenthaltsrechtlichen Voraussetzungen. In einigen Bundesländern bieten Berufsschulen Vollzeitbildungsgänge an, in denen Schulabschlüsse nachgeholt werden können (ebd., S. 37 f.).

Die Angebote zur Berufsvorbereitung in den einzelnen Bundesländern sind unterschiedlich gestaltet. Ein wesentliches Unterscheidungskriterium besteht vor allem darin, dass es in einigen Bundesländern ein Angebot gibt, das sich explizit an Migrantinnen und Migranten richtet, wie z. B. in Bayern, Thüringen, Niedersachsen oder auch Hamburg. So wurden beispielsweise an Hamburger Berufsbildenden Schulen für jugendliche Migranten grundsätzlich zwei Bildungsgänge etabliert: für Schülerinnen und Schüler mit ungesichertem Aufenthaltsstatus wird ein zweijähriger Vorbereitungskurs (VJ-M) angeboten, der das Ziel verfolgt, eine möglichst umfassende Handlungskompetenz zu entwickeln, die eine erste gesellschaftliche Orientierung ermöglicht. Der Personenkreis mit einem gesicherten Aufenthaltsstatus soll dagegen in einer zweijährigen Berufsvorbereitung gezielt auf die notwendigen Kompetenzen für eine Berufsausbildung in einem bestimmten Berufsfeld (BVJ-M) vorbereitet werden. In Niedersachsen können beispielsweise Berufsbildende Schulen für schulpflichtige Jugendliche ohne Ausbildungsvertrag ein Berufsvorbereitungsjahr in der Sonderform für Aussiedlerinnen und Aussiedler sowie Ausländerinnen und Ausländer (BVJ-A) anbieten (Weiser, 2013, S. 18). In Bundesländern wie z. B. in Mecklenburg-Vorpommern, Rheinland-Pfalz oder im Saarland findet keine für Migranten spezifische Berufsvorbereitung statt (Deutscher 


\section{Essbiografisches Lernen}

Bildungsserver, 2015), in einigen Bundesländern wiederum liegen solche Angebote über freie Träger vor, wie z. B. in Berlin. Vor dem Hintergrund der rasant ansteigenden Zahl von Flüchtlingen, reagieren aktuell allerdings viele Bundesländer kurzfristig mit sogenannten Willkommens-, Vor-, Übergangs- oder Vorbereitungsklassen (Schuler \& Sadigh, 2015). Diesbezüglich wird es sicherlich auch weiterhin neue Entwicklungen geben.

\section{Bezugspunkte für ein didaktisches Konzept für minderjährige Flüchtlinge in der Berufsorientierung}

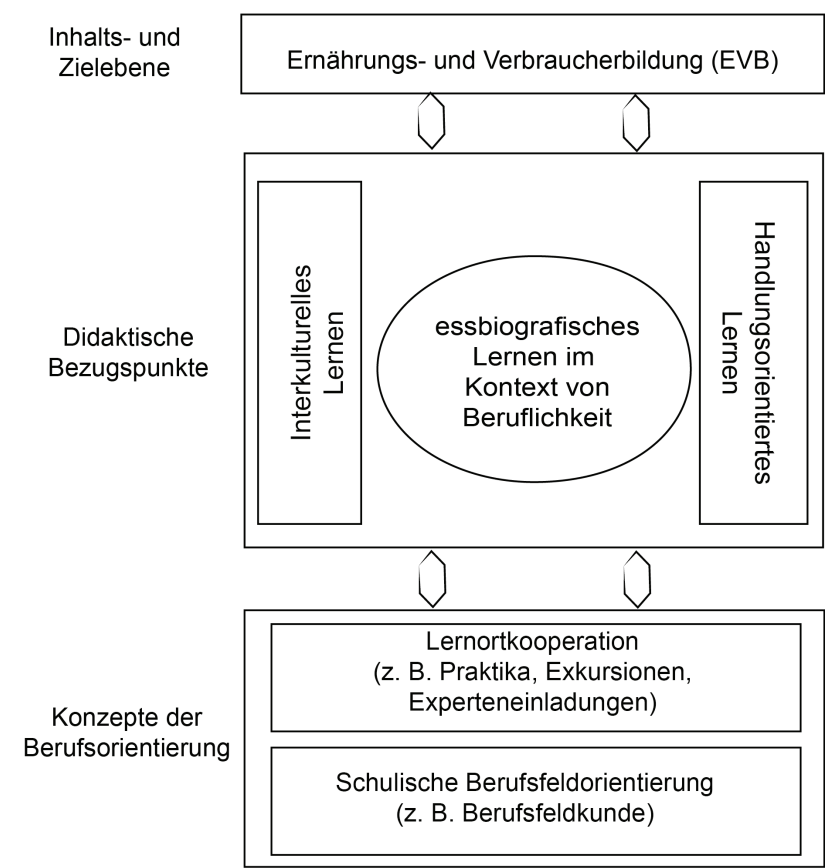

Abb. 1: Architektur des didaktischen Konzepts für minderjährige Flüchtlinge in der Berufsvorbereitung (Quelle: eigene Darstellung)

Das vorgeschlagene didaktische Konzept verfolgt den Ansatz einer fächerübergreifenden Ernährungs- und Verbraucherbildung, wobei die Methodik des essbiografischen Lernens erweitert um gastgewerbliche und hauswirtschaftliche Fragestellungen (Abschnitt 2.1) in Verbindung mit handlungsorientiertem Lernen (Abschnitt 2.2) unter Berücksichtigung von Prinzipien interkulturellen Lernens angewandt wird (Abschnitt 2.3). Weitere Impulse auf der Inhalts- und Zielebene können bestehende Konzepte und Projekte der Ernährungs- und Verbraucherbildung (EVB) liefern (Kapitel 3). 
Ein solcher Referenzrahmen (s. Abbildung 1) kann einen Beitrag für eine erfolgreiche Ausbildungs- und Arbeitsmarktintegration von minderjährigen Flüchtlingen leisten. Unter besonderer Berücksichtigung des essbiografischen Lernens soll die Möglichkeit geschaffen werden, Handlungskompetenzen zu erlangen, die neben dem deutschen Spracherwerb und der Alltagsorientierung auch zu einer gastgewerblichen Berufsfeldorientierung führen. Das Konzept, ergänzt um eine Lernortkooperation im Berufsfeld, kann zusätzliche arbeitsmarktintegrative Impulse setzen. Auf diese Weise können die Chancen der minderjährigen Flüchtlinge auf eine erfolgreiche Ausbildungs- und Arbeitsmarktintegration erhöht werden.

\subsection{Essbiografisches Lernen als Basis des didaktischen Konzepts}

Die Methode des essbiografischen Lernens stellt eine Herangehensweise innerhalb des biografischen Lernens dar. Biografisches Lernen zeichnet sich dadurch aus, dass unterrichtliche Prozesse mit und an individuellen Biografien ausgerichtet werden (Bender, 2013, S. 71). Das Potenzial von biografischem Lernen liegt darin, dass ,die Reflexion von Biografien - der eigenen und der anderer Menschen - für Lernende einen wesentlichen Zugang zu fachlichen Inhalten, Werten und Bewertungen, Haltungen und Handlungen bietet. Ein derartiger biografieorientierter $\mathrm{Zu}-$ gang kann die Fähigkeit und die Bereitschaft zu Reflexion und Selbstreflexion sowie den Umgang mit anderen entscheidend fördern" (Methfessel, 2003, S. 32).

Essbiografisches Lernen ist eine Methode, bei der der Zusammenhang zwischen der Biografie sowie der Entstehung von Essmustern und Herkunft von Essgewohnheiten als Teil von Konsum und Lebensstil beleuchtet wird: „Essmuster und Gewohnheiten entstehen im Lebenslauf. Als Methode verstanden, analysiert und beschreibt die Essbiografie Herkunft und Entwicklung dieser Muster und Gewohnheiten" (Lehrplankommission Verbraucherbildung, 2009).

Beim essbiografischen Lernen bilden Einflüsse auf die eigene Esskultur und auf das individuelle soziale Umfeld den Ausgangspunkt für eine inhaltliche Betrachtung eines Themas. Beim Arbeiten mit Essbiografien wird folglich stets auch eine gewisse Wechselwirkung zwischen Individuum und Gesellschaft thematisiert, da individuelle Erfahrungen immer auch Hinweise auf allgemein-gesellschaftliche Zusammenhänge geben und dabei in einem ganz wesentlichen Zusammenhang zu der sozialen Schicht oder der Kultur, in der ein Mensch aufwächst, stehen (Miethe, 2014, S. 19). Begründet in der Tatsache, dass alle Menschen, unabhängig von ihrer Herkunft, ihrer Kultur oder ihrem Bildungsstand Erfahrungen mit Essgewohnheiten haben, wird ein großer Vorteil des essbiografischen Lernens deutlich: insbesondere bei stark heterogenen Lerngruppen kann über die eigene Essbiografie ein allgemeingültiger Zugang eröffnet werden, so auch bei Flüchtlingen. Die Methode des essbiografischen Lernens ermutigt die Lernenden, sich mit individuellen Vor- 


\section{Essbiografisches Lernen}

lieben, Prägungen und Abneigungen im Rahmen ihrer Essgewohnheiten auseinanderzusetzen. Aufgrund dessen kann der kulinarische Entwicklungsprozess kritisch reflektiert und als Einstieg für weitere Lernprozesse genutzt werden, wie z. B. zum Spracherwerb, für die Ausbildung mathematischer Fähigkeiten etc. Den Lernenden kann durch die Betrachtung und Bearbeitung von unterschiedlichen Essbiografien bewusst gemacht werden, dass jeder Mensch über eine einzigartige und individuelle Essbiografie verfügt, die es zu tolerieren und zu akzeptieren gilt. Dabei geht es grundsätzlich nicht ,um die Rekonstruktion von Fakten bzw. dessen Realitätsgehalt, als vielmehr um das Verstehen des „Eigen-Sinns“ biografischer Äußerungen“ (Miethe, 2014, S. 21).

Ein so konstruiertes Bildungskonzept ermöglicht es den Lernenden, umfangreiche kommunikative und kulturelle Zusammenhänge zu erleben und zu erfahren, die sich bei einer Ess- und Mahlzeitengestaltung sowie der damit in Zusammenhang stehenden Nahrungszubereitung ergeben. Für einen so ausgerichteten Lernprozess sind Reflexionen über die eigenen Zugänge zum Essen, eine Sensibilisierung der Selbstwahrnehmung und eine Auseinandersetzung mit Erinnerungen und Emotionen in Bezug auf Ernährung grundlegend (Bender, 2013, S. 72).

Für den Erfolg biografischen Lernens und insbesondere auch des essbiografischen Lernens ist die professionelle Anleitung durch den Lehrenden besonders entscheidend, da bei einem unprofessionellen Umgang ,eher Schaden als Nutzen angerichtet werden kann" (Miethe, 2014, S. 153). Schließlich können durch das biografische Lernen sehr persönliche Dinge der Lernenden zu Tage kommen und im Kontext von Essbiografien auch traumatische oder zumindest belastende Erfahrungen aufgedeckt werden. Bei Flüchtlingen könnten dies z. B. Erfahrungen mit Nahrungsmittelknappheit oder gar Hunger sein.

Um insbesondere diese psychologische bzw. therapeutische Komponente des essbiografischen Lernens mit den Lernenden zu relativieren, für die Lehrkräfte in der Regel nicht entsprechend qualifiziert sind, kann es zielführend sein, im Kontext des essbiografischen Lernens stärker die Beruflichkeit in den Mittelpunkt zu stellen. Dies entspräche dann auch den Konzepten der Berufsorientierung, wenn auf diese Weise berufliche Interessen bei den Flüchtlingen angebahnt würden. D. h. für das Berufsfeld Ernährung und Hauswirtschaft müssen Fragen der gastronomischen und hauswirtschaftlichen Fachlichkeit aufgegriffen und methodisch mit (ess)biografischem Lernen ausgefüllt werden. Das essbiografische Lernen würde damit erweitert um eine gastgewerbliche und hauswirtschaftliche Dimension. Auf diese Weise wird der Ansatz des essbiografischen Lernens berufspädagogisch angepasst und es kann gelingen, die biografische Methode mit der Berufspropädeutik zu verknüpfen. Persönliche Aspekte rücken dadurch in den Hintergrund; im Vordergrund steht die Beruflichkeit. Für den vorliegenden Beitrag gilt damit dieses erweiterte Verständnis von essbiografischem Lernen, wobei an dem Begriff selbst festgehalten wird. 
Im Kontext eines solch erweiterten Verständnisses des essbiografischen Lernens könnten - unter zu Hilfenahme von Methoden biografischen Lernens (siehe zur Systematisierung von Methoden der Biografiearbeit (Miethe, 2014, S. 41 ff.) Erinnerungen, Erfahrungen, Emotionen und auch Einstellungen der Lernenden aufgegriffen werden zu Gastlichkeit und Gastfreundschaft, Festlichkeit, gutem Service (in der Gastronomie oder Hotellerie), benötigten Geräten und Utensilien zur Mahlzeitenzubereitung, der Ausstattung einer (guten) Küche, Beschaffungswege von Lebensmitteln, Zubereitung und Verarbeitung von Lebensmitteln, Aufgaben bei der Mahlzeitenzubereitung u. v. m. Diese und weitere Fragestellungen müssten im Rahmen einer weiteren Ausarbeitung und Erprobung des vorgeschlagenen Konzeptes weiterentwickelt und validiert werden.

Eine didaktische Herausforderung besteht darin, solche Lernsituationen zu schaffen, die Vergleiche und Erweiterungen der subjektiven Einsichten von Lernenden mit anderen Biografien, Expertenwissen und Fachwissen ermöglichen. Es muss gelingen, sowohl „Subjektives“ als auch „Allgemeines“ zum Lerngegenstand zu machen, so dass eine lernunterstützende Spannung zwischen Fachwissen und individuellen Erfahrungen aufgebaut werden kann. Expertenwissen stellt an berufsbildenden Schulen einerseits die Lehrkraft selbst zur Verfügung. Es können aber auch Lernortkooperationen mit Betrieben genutzt werden, um beispielsweise Praktika anzubahnen, Experten einzuladen oder Exkursionen durchzuführen.

Das oben aufgezeigte Verständnis von essbiografischem Lernen für die Arbeit mit Flüchtlingen mit dem Ziel der Berufsorientierung kann sich grundsätzlich an den vier Phasen des biografischen Lernens nach Methfessel (2003) orientieren:

Tab. 1: Phasen des biografieorientierten Lernens mit Beispielen im Kontext von Berufsorientierung im Berufsfeld Ernährung und Hauswirtschaft (Methfessel, 2003, S. 39, Bender, 2013, S. 73 f.)

\begin{tabular}{c|l|l}
\hline \multicolumn{1}{c|}{ Phase } & \multicolumn{1}{|c}{ Beschreibung } & $\begin{array}{l}\text { Beispiele essbiografischen Lernens } \\
\text { im Kontext von Berufsorientierung } \\
\text { im Berufsfeld Ernährung und } \\
\text { Hauswritschaft }\end{array}$ \\
\hline 1) Erinnern & $\begin{array}{l}\text { Lernende werden methodisch darin } \\
\text { unterstützt, sich an ausgewählte } \\
\text { inhaltlich relevante Situationen ihrer } \\
\text { Biografie zu erinnern. Es gilt, dass } \\
\text { diese Erlebnisse möglichst lebendig } \\
\text { zu vergegenwärtigen sind. }\end{array}$ & $\begin{array}{l}\text { Mögliche Instrumente: Essgeschirr, } \\
\text { Küchenutensilien, Lebensmittel aus } \\
\text { kulturell unterschiedlichen Bereichen, } \\
\text { persönliche Aufzeichnungen, Fotos } \\
\text { von Mahlzeitensituationen } \\
\text { Rollenspiele zu Mahlzeitensituationen } \\
\text { in familiären und institutionellen }\end{array}$ \\
\hline 2) Aneignen & $\begin{array}{l}\text { Die Lernenden symbolisieren die } \\
\text { ausgewählten Situationen durch } \\
\text { Erzählungen, Zeichnungen, Colla- } \\
\text { gen, Rollenspiele etc. Auf diese } \\
\text { Weise lernen sie damalige und } \\
\text { aktuelle Erlebnisse (Gefühle, Phan- } \\
\text { tasien und subjektive Bedeutungen) }\end{array}$ & $\begin{array}{l}\text { Esstagebücher oder Essprotokolle } \\
\text { Wege der Beschaffung von Lebens- } \\
\text { mitteln aufzeigen } \\
\text { Skizzen zu Ausstattung von Küchen }\end{array}$ \\
\hline
\end{tabular}




\section{Essbiografisches Lernen}

\begin{tabular}{|c|c|c|}
\hline & $\begin{array}{l}\text { mit der jeweiligen Situation zu } \\
\text { verbinden. Dieses Bewusstmachen } \\
\text { lässt Erlebnisse zu Erfahrungen } \\
\text { transformieren. }\end{array}$ & \\
\hline 3) Verarbeiten & $\begin{array}{l}\text { Die Lernenden reflektieren und } \\
\text { interpretieren die Erfahrungen in } \\
\text { Kleingruppen. Es erfolgt ein Aus- } \\
\text { tausch mit anderen Erfahrungen, } \\
\text { Biografien, weitergehenden Infor- } \\
\text { mationen und Expertenwissen. Auf } \\
\text { diese Art erarbeiten sich die Ler- } \\
\text { nenden allgemeinere thematische } \\
\text { Zugänge. }\end{array}$ & $\begin{array}{l}\text { Es geht auch um Themenfelder einer } \\
\text { modernen Ernährungs- und Konsum- } \\
\text { bildung (s. u.) und dem Herausarbei- } \\
\text { ten von Unterschieden und Gemein- } \\
\text { samkeiten im Ernährungsverhalten } \\
\text { verschiedener Personen und Gruppen. } \\
\text { Expertengruppen zu unterschiedliche } \\
\text { Entwicklungen von traditionellen } \\
\text { Gerichten. } \\
\text { Fishbowl zur Entwicklung und Durch- } \\
\text { führung von Feste. } \\
\text { Erfahrungsaustausch zur familiären } \\
\text { Aufgabenverteilung im Rahmen der } \\
\text { Vorbereitung auf große Familienfeste. }\end{array}$ \\
\hline $\begin{array}{l}\text { 4) Veröffentli- } \\
\text { chen }\end{array}$ & $\begin{array}{l}\text { Abschließend werden die Gruppen- } \\
\text { ergebnisse außerhalb der Kleingrup- } \\
\text { pen präsentiert und Erfahrungen } \\
\text { aufgezeigt. }\end{array}$ & $\begin{array}{l}\text { Präsentation in unterschiedlichen } \\
\text { Formen, z. B. als Marktplatz (Plakaten } \\
\text { und Kleinpräsentationen). } \\
\text { Klassen-, Schul- oder Stadtteilfest, bei } \\
\text { dem die unterschiedliche kulinarische } \\
\text { Entwicklung deutlich wie. }\end{array}$ \\
\hline
\end{tabular}

Inwieweit das Verständnis essbiografischen Lernens Anknüpfungspunkte und Überschneidungen zum Verständnis der interkulturellen Bildung aufweist, wird im folgenden Abschnitt weiter herausgearbeitet.

\subsection{Interkulturelles Lernen als Ergänzung des didaktischen Konzepts}

Deutschland entwickelt sich zu einer kulturell pluralistischen Gesellschaft mit einem steigenden Anteil an ausländischen Mitbürgern, die auf Dauer in Deutschland leben und arbeiten wollen. Dies ist auch mitbedingt durch die derzeitig hohe Zahl an Menschen, die als Flüchtlinge nach Deutschland kommen. Die Entwicklung erfordert von den Deutschen Fähigkeiten und Kenntnisse, um mit den ausländischen Mitbürgern friedlich und sich gegenseitig bereichernd zusammenzuleben und zu arbeiten. Es ist entscheidend, Kenntnisse über die jeweils anderen Kulturen zu erlangen, um Missverständnisse und Konflikte zu vermeiden (Thomas, 2006, S. 115). Diese Fähigkeiten und Kenntnisse sind aber auch bei Flüchtlingen selbst zu fördern, da sie ebenfalls aus unterschiedlichen Kulturkreisen kommen, sie gerade in der ersten Phase ihres Aufenthaltes häufig unter sich wohnen und leben. Interkulturelle Kompetenzen können ihnen dabei helfen, sich in der deutschen Gesell- 


\section{Essbiografisches Lernen |}

schaft einzufinden und zu integrieren. Auch in den Berufsschulklassen, in denen die jugendlichen Migranten unterrichtet werden, findet sich eine in Bezug auf die Kulturen heterogene Gruppe. Die notwendigen Fähigkeiten und Kenntnisse können in dem Begriff Interkulturelle Handlungskompetenz zusammengefasst werden (Thomas, 2006, S. 116). Interkulturelle Handlungskompetenz ist das Ergebnis eines Lern- und Entwicklungsprozesses, an dessen Anfang die Bereitschaft zur Auseinandersetzung mit fremden kulturellen Orientierungssystemen sowie eine Grundhaltung kultureller Wertschätzung stehen. Interkulturelle Kompetenz basiert auf handlungstheoretischen Annahmen und bietet insofern auf einer theoretischen Ebene Anknüpfungspunkte zum handlungsorientierten Lernen (s. Abschnitt 3.3).

Caspari (2007, S. 71) unterteilt interkulturelle (Handlungs)kompetenz in:

1. Erwerb von Einstellungen, u. a. die Bereitschaft, mit begrenzten sprachlichen Mitteln Kommunikation zu wagen und offen auf Fremde zuzugehen und sie zu tolerieren.

2. Erwerb von Fähigkeiten, d. h. die Fähigkeit, eigene und fremde Äußerungen und Verhaltensweisen sowohl als kulturabhängig als auch als individuell zu begreifen und mit den eigenen eingeschränkten sprachlichen Kompetenzen konstruktiv umzugehen.

3. Erwerb von Wissen, sowohl von Wissen über Kommunikation als auch kulturspezifisches Wissen, als Hilfe zur adäquaten Deutung kommunikativer Äußerungen sowie zur Prävention beziehungsweise Klärung und Aufklärung von Missverständnissen.

Korrespondierend zum Begriff der beruflichen Handlungskompetenz wäre zu überlegen, die Kompetenzdimensionen der beruflichen Handlungskompetenz (Fach-, Sozial- und Humankompetenz, Lern- und Methodenkompetenz sowie kommunikative Kompetenz) im Sinne interkultureller Handlungskompetenz auszugestalten.

Die Kultusministerkonferenz hat erstmals 1996 „Empfehlungen zur Interkulturellen Bildung und Erziehung in der Schule" formuliert und diese im Dezember 2013 in einer überarbeiteten Fassung spezifiziert. In diesem Orientierungsrahmen wird die interkulturelle Bildung als eine Querschnittsaufgabe für Schulen festgeschrieben. Demnach haben auch berufsbildende Schulen die Aufgabe, allen Lernenden, unabhängig von ihrer Herkunft eine umfassende Teilhabe an Bildung und damit den größtmöglichen Bildungserfolg zu gewährleisten. Eine erfolgreiche Gestaltung von Integrationsprozessen trägt in der Folge zu einem friedlichen und demokratischen Zusammenleben bei, in dem überdies eine Orientierung für verantwortungsbewusstes Handeln in einer globalisierten Welt vermittelt wird (KMK, 2013, S. 2). Berufsbildende Schulen sind folglich gefordert, Handlungskompetenzen für den Umgang mit Vielfalt zu entwickeln und umzusetzen. Das Fördern interkultureller Kompetenzen für ein verantwortungsvolles Handeln in einer pluralistischen und global vernetzten Gesellschaft beschränkt sich jedoch nicht allein auf 


\section{Essbiografisches Lernen}

eine Auseinandersetzung mit anderen Sprachen und Kulturen. Darüber hinaus sollen die Lernenden in die Lage versetzt werden ,sich selbstreflexiv mit den eigenen Bildern von Anderen auseinander und in Bezug zu setzen sowie gesellschaftliche Rahmenbedingungen für die Entstehung solcher Bilder zu kennen und zu reflektieren" (ebd., S. 2). Für die systematische und interkulturelle Entwicklung von Schulen stellt die KMK (2013, S. 3 ff.) folgende Grundsätze auf:

1. Schule nimmt Vielfalt zugleich als Normalität und als Potenzial für alle wahr.

2. Schule trägt zum Erwerb interkultureller Kompetenzen im Unterricht aller Fächer und durch außerunterrichtliche Aktivitäten bei.

3. Schule ist zentraler Ort für den Erwerb bildungssprachlicher Kompetenzen.

Diesen Grundsätzen kann durch eine fächerübergreifende Ausrichtung der Lerninhalte auf die Methodik des essbiografischen Lernens, vor allem bei der Gestaltung von Lehr-/Lernprozessen für Flüchtlinge entsprochen werden.

\subsection{Handlungsorientiertes Lernen als Ergänzung des didaktischen Konzepts}

Das Konzept der Handlungsorientierung ist seit der Einführung der Lernfeldsystematik in den 90er Jahren in der beruflichen Bildung verankert. Es führt eine Vielzahl von unterschiedlichen wissenschaftlichen Disziplinen und Ansätzen zusammen und gilt nicht zuletzt als ein didaktisch-methodisches Grundprinzip in der Benachteiligtenförderung (BMBF, 2005, S. $91 \mathrm{ff}$.). Ziel dieses Ansatzes in der Berufsvorbereitung ist es, den benachteiligten Jugendlichen die Möglichkeit zu geben, sich aktiv und handlungswirksam wahrnehmen zu können (BIBB, 2007, S. 13). Vor diesem Hintergrund stellt das Konzept der Handlungsorientierung auch in dem hier vorgelegten didaktischen Konzept eine elementare Stellschraube dar, an der sich der Unterricht mit den Flüchtlingen neben dem essbiografischen und dem interkulturellen Lernen orientieren sollte. Unterschiedliche wissenschaftliche Disziplinen und Ansätze zur theoretischen Fundierung (z. B. Tramm, 1996; Schelten, 2000; Dubs, 1995) legen Merkmale handlungsorientierten Unterrichts vor. Allen Ansätzen gemeinsam ist die Forderung nach einer aktiven Auseinandersetzung des Lernenden mit dem Lerngegenstand bzw. -inhalt, um hierüber Wirklichkeit sowie Erfahrungs- und Handlungsspielräume zu schaffen (Beck, 1996, S. 55). Zielgemäß geht es um die Vermittlung von theoretischen Voraussetzungen für das Handeln, die sich in der Berufsvorbereitung teilweise bereits auf das Handeln in der Berufspraxis bezieht. 
Essbiografisches Lernen |

\subsection{Verknüpfungsmöglichkeiten der didaktischen Bezugspunkte}

Eine Verknüpfung von essbiografischem, interkulturellem und handlungsorientiertem Lernen bietet ein großes Potenzial in Berufsvorbereitungsklassen mit bzw. für minderjährige Flüchtlinge bzw. Migrantinnen und Migranten. Möglichkeiten zur Ausgestaltung von Lehr- Lernarrangements entstehen so beispielsweise durch eine Betrachtung und Bearbeitung von spezifischen Esskulturen innerhalb dieser Klassen. Hierdurch kann verdeutlicht werden, dass Esskulturen u. a. durch Globalisierung, Migration und Heterogenität beeinflusst werden. Eine gezielte Auseinandersetzung mit dieser Thematik wird gesundheitliche, geografische, ökonomische, soziale, kulturelle, migrationsspezifische, religiöse, politische, psychische und physische Aspekte hervor bringen. Hieraus ergeben sich zahlreiche Widersprüche und Herausforderungen für das individuelle Handeln und Essverhalten, die für eine Entwicklung von entwicklungs- und lernförderlichen Aufgaben im interkulturellen Kontext nutzbar gemacht werden können. Aufgezeigte Gemeinsamkeiten und Unterschiede führen so zu einer offenen Kommunikation über die vielfältigen Formen von Essen und Ernährung. Diese Form der Auseinandersetzung mit der eigenen Essbiografie und der von Mitschülerinnen und Mitschülern schafft Gelegenheiten, Lerninhalte bzw. Lerngegenstände wirklichkeitsnah und auf individuellen Erlebnissen und Erfahrungen basierend in unterrichtliche Lehr-/Lernarrangements einzubeziehen. Eine vor diesem Hintergrund gestaltete Lernsituation bietet die aktive Beschäftigung mit subjektiven Erfahrungen und verdeutlicht überdies die Verbindungen zum handlungsorientierten Lernen.

\section{Bezugspunkte auf der Inhalts- und Zielebene}

Weitere Anregungen für das vorgeschlagene didaktische Konzept auf der Inhaltsund Zielebene können u. a. Konzepte der Ernährungs- und Verbraucherbildung liefern, die sich seit Ende der 1980er Jahre zu den Schwerpunkten Nahrung, Essen, Ernährung und Konsum auf europäischer und deutscher Ebene entwickelt haben. Ernährungs- und Verbraucherbildung zielt letztendlich auf die Förderung von Ernährungs-, Gesundheits- und Konsumkompetenz ab. Menschen sollen dabei unterstützt werden, „eine persönlich sinnvolle Lebensweise durch gesunde Lebensführung aufzubauen“ (Heindl \& Plinz-Wittorf, 2010, S. 6), wobei sowohl individuelle als auch soziale, ökologische und ökonomische Handlungsweisen entfaltet werden sollen (ebd.). In Deutschland wurde das europäische Kerncurriculum zur Ernährungsbildung auf der Grundlage umfassender Analysen zur Ernährungserziehung in deutschen allgemeinbildenden Schulen um Aspekte der Verbraucherbildung erweitert - festgelegt im viel beachteten REVIS-Curriculum mit entsprechenden Bildungszielen, Kompetenzen, Themen und Inhalten (aid infodienst Ernährung, 


\section{Essbiografisches Lernen}

2013, S. 10). REVIS war von 2003 bis 2005 ein Forschungs- und Entwicklungsprojekt zur Reform der Ernährungs- und Verbraucherbildung in allgemeinbildenden Schulen (Universität Paderborn, 2005). Ein Bildungsziel der EVB bezieht sich auf die Fähigkeit der Schülerinnen und Schüler ihre eigene Essbiografie reflektiert und selbstbestimmt gestalten zu können und die Kompetenz zu erlangen, ,sich mit den Einflussfaktoren, Begrenzungen und Gestaltungsalternativen der individuellen Essweise auseinanderzusetzen“ (ebd., o. S.).

Auch das Projekt „Food Literacy“ (Laufzeit 2004-2007) kann der Ernährungsund Verbraucherbildung zugeordnet werden, wobei die vorliegenden Erkenntnisse, Erfahrungen und Materialien (Schnögl et al., 2006, S. 37 ff.) insbesondere für den außerschulischen Bereich und die Zielgruppe der Erwachsenen entwickelt wurden. Der Begriff , literacy“ als Ausgangspunkt der Rahmenkonzeption der Pisa-Studien stellt eine Grundbildung dar, bei der es um die Fähigkeiten der Lernenden geht, „Kenntnisse und Fertigkeiten in einer Vielzahl von Situationen anzuwenden und zu analysieren, logisch zu denken und in effektiver Weise zu kommunizieren" (Pisa Konsortium Deutschland, 2005, S. 16). Food Literacy, verstanden als die „Fähigkeit, den Ernährungsalltag selbstbestimmt, verantwortungsbewusst und genussvoll zu gestalten" (Büning-Fesel, 2008, S. 2), soll bei den Lernenden Empowerment (im Sinne von Selbstbestimmung beim Ernährungshandeln), Entscheidungskompetenz (z. B. im Umgang mit Überangebot an Lebensmitteln) und Basiskompetenzen (z. B. Zubereitung von Lebensmitteln aus frischen, saisonalen Produkten) fördern (aid infodienst, 2014, S. 12). Es bietet vor allem Potenzial für sozial Benachteiligte und bildungsferne Personen als Zielgruppe, da Gegenstand die Auseinandersetzung mit einem alltagsbezogenen Themengebiet ist. Zudem können vor allem multikulturell zusammengesetzte Gruppen von einem interkulturellen Austausch über die kulturellen Hintergründe sowohl sprachlich als auch emotional profitieren. Individuelle und kulturell verschiedenartige Lebensgeschichten bieten die Grundlage für eine themenspezifische Bearbeitung von Bildungsinhalten (ebd., S. 9).

Warum aber sollten in einem didaktischen Konzept für Flüchtlinge in der Berufsorientierung auch Inhalte und Zielsetzungen der Ernährungs- und Verbraucherbildung aufgegriffen werden?

Gerade jugendliche, minderjährige Flüchtlinge befinden sich in der Entwicklungsphase des Heranwachsenden bzw. jungen Erwachsenen, die Entscheidungen in Bezug auf Berufswahl, Lebensplanung, Erlangung einer wirtschaftlichen und sozialen Eigenständigkeit sowie der Gründung eines eigenen Haushalts und ggf. einer eigenen Familie treffen müssen (Gemballa \& Kettschau, 2011, S. 21). Auch wenn die unbegleiteten minderjährigen Flüchtlinge in der Regel Unterstützung und Orientierung in der Jugendhilfe bis zu ihrer Volljährigkeit erfahren, ist es gerade für diese jungen Menschen von großer Bedeutung, dass sie darin unterstützt und bestärkt werden, die genannten Lebensbereiche selbständig und eigenverantwortlich zu gestalten. Dies kann bei den Flüchtlingen, die eine Berufsschule besuchen, 
zusätzlich durch eine entsprechende EVB gefördert werden, wobei sich die Zielsetzungen für die Berufsorientierung mit der Methode des essbiografischen Lernens und den Zielen und Anliegen des interkulturellen Lernens verknüpfen lassen.

\section{Umsetzungserfahrungen und Ausblick}

Die Idee zum vorgeschlagenen Konzept ist im Rahmen der Umsetzung des 2012 neu eingeführten BVJ-M (s. oben) an der Beruflichen Schule für Wirtschaft und Steuern in Hamburg entstanden. Im Schwerpunkt verfolgt dieser Bildungsgang den Aufbau und die Förderung der deutschen Sprachkompetenz bei den Flüchtlingen. Neben der beruflichen Vorbereitung haben die Schülerinnen und Schüler außerdem die Möglichkeit, einen ihren Leistungen entsprechenden allgemeinbildenden Schulabschluss zu erwerben. Diese Ziele sollen einerseits durch ein praxisorientiertes Projekt im Fach Arbeitslehre und andererseits durch Unterricht in den Fächern Berechnungen, Sprache und Kommunikation, Wirtschaft und Gesellschaft, Englisch und Sport erreicht werden.

Erste Erfahrungen mit dem essbiografischen Lernen verknüpft mit interkulturellem und handlungsorientiertem Lernen sind im Fach Arbeitslehre entstanden. Ausgangsidee war, dass die Schülerinnen und Schüler Gerichte aus ihrem Heimatland vorstellen, zubereiten und gemeinsam verzehren. Hierfür bekamen die Schülerinnen und Schüler die Möglichkeit mit einem eigenen Budget eine Küche zu planen, zu beschaffen und zu montieren und mit entsprechenden Küchengeräten und -materialien zu bestücken. Die Schülerinnen und Schüler wählten geeignete Gerichte aus, verschriftlichten und präsentierten Rezepturen und erstellten Einkaufslisten. Sie schlüpften für je einen Tag in die Rolle einer Küchenchefin bzw. eines Küchenchefs, die/der Aufgaben zur Zubereitung des Gerichtes an die anderen Schülerinnen und Schüler delegieren durfte. Hierbei lag ein Schwerpunkt auf der Erprobung von Sozialstrukturen (z. B. Frauen als Chefin), die den Traditionen aus den Herkunftsländern widersprachen. In der Abschlussprüfung wurden u. a. eine Rezeptur und ein Ablaufplan erstellt und in einem praktischen Teil umgesetzt.

Im Rahmen des Projektes konnten Grundlagen des Wirtschaftens handlungsorientiert erarbeitet werden. So wurde beispielsweise die Entstehung von Kaufverträgen und die daraus resultierenden Rechte und Pflichten erlebbar gemacht. Preise und Qualitätsmerkmale der Küchenausstattung wurden recherchiert und bewertet. Gleichzeitig kamen Lerninhalte der Lebensmittelkunde und ernährungsphysiologische Aspekte zum Tragen. Durch die Planung der Küche und der Gerichte sowie die Übernahme der Rolle einer Küchenleitung wurden auch Elemente der Berufsorientierung aufgegriffen. Ausgangspunkt für weitere essbiografische, interkulturelle und handlungsorientierte Lernprozesse könnten beispielsweise auch die Entwicklung eines Restaurantführers für interkulturelle Küche in der neuen 


\section{Essbiografisches Lernen}

Heimatstadt sein oder ein Vermarktungsprojekt, das auf die Herstellung und den Verkauf von Gewürzmischungen oder Ölen abzielt.

Tab. 2: Beispiel für einen Stoffverteilungsplan in der Berufsvorbereitung für Migrantinnen und Migranten im 2. und 3. Schulhalbjahr (Quelle: eigene Darstellung)

\begin{tabular}{|c|c|c|c|}
\hline $\begin{array}{c}\text { Inhalte/ } \\
\text { Schlüsselbegriffe }\end{array}$ & $\begin{array}{l}\text { Methoden/ } \\
\text { Beispiele }\end{array}$ & Leistungskriterien & Fächerübergreifend \\
\hline $\begin{array}{l}\text { Essen und Identität: } \\
\text { soziale, religiöse und } \\
\text { familiäre Einflüsse } \\
\text { kulturelle } \\
\text { Unterschiede } \\
\text { Lebensmittelkunde } \\
\text { Zusammenhang von } \\
\text { Ernährung und } \\
\text { Gesundheit } \\
\text { Selbstwirksamkeit des } \\
\text { Essverhaltens } \\
\text { Verantwortung für } \\
\text { eigenes Handeln } \\
\text { Umgang mit } \\
\text { Konflikten } \\
\text { Lebensplanung und } \\
\text { Gestaltung } \\
\text { individuelle und } \\
\text { gesellschaftliche } \\
\text { Ressourcen } \\
\text { Bedürfnisse, Bedarf, } \\
\text { Budget } \\
\text { ästhetisch-kulinarische } \\
\text { und } \\
\text { zielgruppenspezifische } \\
\text { Speisenzubereitung } \\
\text { Arbeitsteilung, - } \\
\text { planung und - } \\
\text { organisation } \\
\text { Konsum und } \\
\text { Entscheidungsprozesse } \\
\text { Marktmechanismen } \\
\text { und Wirtschaftssystem }\end{array}$ & $\begin{array}{l}\text { „Meine } \\
\text { Essbiografie“ } \\
\text { „Was esse } \\
\text { ich?“” } \\
\text { „Die ganze } \\
\text { Welt im } \\
\text { Einkaufskorb“ } \\
\text { Projektarbeit, } \\
\text { z.B.: } \\
\text { Herstellen und } \\
\text { Vermarkten } \\
\text { traditioneller } \\
\text { Gewürz- oder } \\
\text { Ölvariationen } \\
\text { Gestalten eines } \\
\text { Flyers über } \\
\text { interkulturelle } \\
\text { Rezepturen } \\
\text { Zubereitung } \\
\text { herkunfts- } \\
\text { bezogener } \\
\text { Gerichte }\end{array}$ & $\begin{array}{l}\text { Kriterien geleitete und } \\
\text { prozess-begleitende } \\
\text { Beobachtungen } \\
\text { Präsentation eines } \\
\text { Ernährungsplakates } \\
\text { Projektpräsentation } \\
\text { Kriterien geleitete und } \\
\text { prozessbegleitende } \\
\text { Beobachtungen }\end{array}$ & $\begin{array}{l}\text { Berechnungen: } \\
\text { Rezepte } \\
\text { Kalkulationen } \\
\text { Warenanforderung } \\
\text { Nährwert-berechnungen } \\
\text { SuK: } \\
\text { Gebrauchstexte } \\
\text { Präsentationen } \\
\text { integrative Grammatik } \\
\text { Sprachverwendung } \\
\text { WuG: } \\
\text { Marktformen } \\
\text { Angebot und Nachfrage } \\
\text { Wirtschaftskreislauf } \\
\text { Marketingaspekte } \\
\text { Engl.: } \\
\text { Vokabelarbeit } \\
\text { Rezepte, } \\
\text { Zubereitungstexte } \\
\text { Sport: } \\
\text { Fit halten } \\
\text { Ernährung und Sport als } \\
\text { notwendiges Wertepaar }\end{array}$ \\
\hline
\end{tabular}




\section{Essbiografisches Lernen |}

Weitreichende Anregungen und methodische Umsetzungsideen bieten die oben aufgeführten Materialien und Konzepte zur EVB. Zudem wurde insgesamt deutlich, dass ein fächerübergreifendes Konzept für das Projekt förderlich gewesen wäre, um die Inhalte - so wie auch im Lernfeldkonzept angedacht - aufeinander zu beziehen und miteinander zu verschränken.

\section{Literatur}

aid infodienst (2014). Essen als Thema in der Erwachsenenbildung. Food Literacy. (2. Aufl., S. 5-63). Bonn: aid.

aid infodienst Ernährung, Landwirtschaft, Verbraucherschutz e. V. (Hrsg.). (2013). Ernährungs- und Verbraucherbildung im Unterricht. Bonn: aid.

Ang-Stein, C. (2015). Interkulturelles Training. Systematisierung, Analyse und Konzeption einer Weiterbildung. Berlin: Springer VS.

Beck, H. (1996). Handlungsorientierung des Unterrichts. Anspruch und Wirklichkeit im betriebswirtschaftlichen Unterricht. Darmstadt.

Bender, U. (2013). Ernährungs- und Konsumbildung. Perspektiven und Praxisbeispiele für den Hauswirtschaftsunterricht. Fachdidaktische Entwicklungen in Deutschland, Österreich und der Schweiz. Bern: Schulverlag.

BMBF (2005). Berufliche Qualifizierung Jugendlicher mit besonderem Förderbedarf. Benachteiligtenförderung. Berlin: Druck Buch.

Bolten, J. (2010). In K. Götz (Hrsg.), Interkulturelles Lernen/Interkulturelles Training. München und Mering: Rainer Hamp.

Büning-Fesel, M. (2008). Food Literacy: Die Förderung von Selbstbestimmung und Entscheidungskompetenz im Ernährungshandeln.

[www.bwpat.de/ht2008/ft04/buening-fesel_ft04-ht2008_spezial4.pdf].

BIBB Bundesinstitut für Berufsbildung (Hrsg.). (2007). Aktuelle Tendenzen in der schulischen Berufsvorbereitung. Bonn.

Bundesfachverband UMF e. V. (2015). Über 45.000 unbegleitete minderjährige Flüchtlinge in Deutschland. [http://www.bumf.de].

Caspari, D. (2007). Didaktisches Lexikon. Landeskunde und Interkulturelles Lernen. Praxis Fremdsprachenunterricht, 5, 70-71.

Deutscher Bildungsserver (2015). Berufsausbildungsvorbereitung: Informationen aus den Bundesländern. [www.bildungsserver.de].

Dubs, R. (1995). Entwicklung von Schlüsselqualifikationen in der Berufsschule. In R. Arnold \& A. Lipsmeier (Hrsg.), Handbuch der Berufsbildung (S. 171-182). Opladen: Leske \& Budrich. [http://dx.doi.org/10.1007/978-3-322-93636-3_13].

Helmrich, R., Zika, G., Kalinowski, M. \& Wolter, M. I. (2012). Engpässe auf dem Arbeitsmarkt: Geändertes Bildungs- und Erwerbsverhalten mildert Fachkräftemangel. Neue Ergebnisse der BIBB-IAB-Qualifikations- und Berufsfeldprojektionen bis zum Jahr 2030. BIBB Report, (6)18, 1-16. 


\section{Essbiografisches Lernen}

Heindl, I. \& Plinz-Wittorf, C. (2010). Schulische Ernährungs- und Verbraucherbildung - Stand der curricularen Entwicklungen. Haushalt \& Bildung, 4, 4-11.

KMK (2013). Interkulturelle Bildung und Erziehung in der Schule. Bonn.

Lehrplankommission Verbraucherbildung (2009). Glossar Lehrplan Verbraucherbildung. [http://lehrplan.lernnetz.de/?wahl_149].

Methfessel, B. (2003). Biografie und Lernen - Allgemeine Überlegungen zu Möglichkeiten und Grenzen in einem lebensweltbezogenen Fach. Haushalt und Bildung, 1, 32-42.

Miethe, I. (2014). Biografiearbeit. Lehr- und Handbuch für Studium und Praxis. (2. Aufl.). Weinheim und Basel: Beltz Juventa.

Pisa Konsortium Deutschland (Hrsg.). (2005). Pisa 2003. Der zweite Vergleich der Länder in Deutschland - Was wissen und können Jugendliche? Münster/New York: Waxmann.

Schelten, A. (2000). Begriffe und Konzepte der berufspädagogischen Fachsprache. Stuttgart: Steiner.

Schnögl. S., Zehetgruber, R., Danninger, S., Setzwein, M., Wenk, R., Freudenberg, M., Müller, C. \& Groeneveld (2006). Schmackhafte Angebote für die Erwachsenenbildung und Beratung. Food Literacy. Handbuch und Toolbox. Wien: Plankopie Eder.

Schuler, K. \& Sadigh, P. (2015). Ab in die Schule, Alima, Bassam und Chipo! [www.zeit.de/gesellschaft/schule/2015-09/schule-fluechtlingskinderjugendliche].

Tramm, T. (1996). Lernprozesse in der Übungsfirma. Rekonstruktion und Weiterentwicklung schulischer Übungsfirmenarbeit als Anwendungsfall einer evaluativ-konstruktiven und handlungsorientierten Curriculumstrategie. Göttingen.

Universität Paderborn (2005). REVIS Modellprojekt. Reform der Ernährungs- und Verbraucherbildung in allgemein bildenden Schulen (2003-2005). Paderborn.

Weiser, B. (2013). Recht auf Bildung für Flüchtlinge. Beilage zum Asylmagazin 11.

\section{Verfasserin und Verfasser}

Prof. ${ }^{\text {in }}$ Dr. Julia Kastrup

Fachhochschule Münster

Institut für Berufliche Lehrerbildung

Leonardo-Campus 7

D-48149 Münster

E-Mail: kastrup@fh-muenster.de
Gorden Steinke, MEd, Referendar

Oscar-Tietz-Schule

Marzahner Chaussee 231

D-12681 Berlin-Marzahn

E-Mail: agp-gorden@web.de 